\title{
Étude des procédés utilisés par les Maures pour empêcher les jeunes animaux de téter
}

\author{
par J. BARON
}

La rudesse du milieu mauritanien impose des limites strictes à la production laitièro des fomclles domestiques. Or, le lait constitue, pour le Maure et sa famille, un aliment d'importance vitale. Il est donc fort compréhensible que les pasteurs maures se soient ingéniés à trouver divers procédés leur permettant de limiter à volonté sa consommation par les jeunes animaux afin d'obtenir pour eux-mêmes la plus grande quantité possible de ce précieux aliment, tout en conciliant cette exigence avec le souci de ménager la santé des femelles laitières et de leurs jeunes.

Bien entendu, ce problc̀me ne so pose pas seulement en Mauritanie et certains des procédés décrits ici ne diffèrent guère de ceux qu'utilisent, un peu partout, tous les éleveurs; par contre, dans d'autres cas, les conditions mêmes de l'élevage en Mauritanie et le génie particulier de la race maure font que les techniques de sevrage mises en œuvre revêtent des aspects singuliers qui, nous semble-t-il, peuvent à des titres divers susciter un certain intérêt.

Pour sevrer temporairement ou définitivement les jounes animaux, l'eleveur maure - comme tous les éleveurs du monde - n'a le choix qu'entre deux sortes de procédés : ou bien séparer les jeunes de leurs mères ou bien établir, soit sur la mamelle de la femelle, soit sur le mufle du petit, un obstacle à la tétée.

\section{I. - Séparation des jeunes.}

Tantôt les jeunes animaux sont placés dans une "zériba 》 (enclos délimité à l'aide de branchages épineux), tantôt ils sont simplement mis à l'attache. On voit souvent, dans les campements maures, de véritables alignements de veaux d'un à deux mois, tous reliés, comme pour une présentation en concours, à une seule longue corde tendue entre deux piquets à $30 \mathrm{~cm}$ du sol. Il en est de même pour les agneaux et les chevreaux. Les veaux déjà forts sont individuellement attachés à des arbres. Lorsque les agneaux sont assez âgés, le Maure qui possède deux troupeaux en profite pour réaliser de façon simple la séparation des mères et de leurs agneaux.
Il Iui suffit pour cela de placer dans le premier troupeau les agneaux du deuxième et vice versa, car ces agneaux ne tetent pas les brebis qui ne sont pas leurs mères. Il évite ainsi d'avoir à former un troupeau uniquement composé de jeunes et pour lequel il lui faudrait rétribuer un berger..

\section{II. - Création d'un obstacle à la tétée.}

1. Au niveau de la mamelle: ces méthodes sont employées chez les chamelles et les petits ruminants.

a) Tout le monde connaît le filet protecteur appliqué sur les mamelles des femelles de dromadaires. Cet appareil a déjà fait l'objet de nombreuses descriptions, nous n'y reviendrons donc pas.

b) Chez les petits ruminants, l'éleveur place une ficelle sur le trayon puis il recouvre le tout de crotte. Le soir, il enlève la ficelle, essuie la mamelle avec la main et laisse téter le jeune animal pendant un certain temps.

$2^{\circ}$ Au niveau du mufle du jeune : on peut classer les procédés en deux groupes, suivant qu'il y a ou non fixation d'un instrument défensif.

a) Procédés sans fixation d'instrument. Ce sont des procédés sanglants et quelque peu répugnants.

- La fente des narines est pratiquée chez les ânons. On fend les deux narines et on laisse pendre les deux lambeaux à droite et à gauche. Si l'on ne fend qu'une narine, l'ânon parvient encore à téter.

- La fente de la langue se fait chez les bovins et quelquefois chez les petits ruminants: Cette méthode barbare consiste à pratiquer une incision de $2 \mathrm{~cm}$ au milieu de la langue dans le sens de la longueur. Cette intervention a lieu au début de l'hivernage et en saison sèche; pendant les derniers mois de l'hivernage où l'herbe verte, bien développée, pourrait se loger facilement dans la plaie, on évite d'utiliser ce procédé qui, même en temps normal, a parfois des conséquences fatales. Il arrive en effet que l'animal succombe soit à l'inanition, soit à une infection gangréneuse de la langue.

- Le «getrane » est la méthode du lambeau de peau coupé sur le chanfrein. L'animal ainsi traité s'appelle « bougetrane ». Cette méthode s'emploie chez les veaux, les ânons et parfois les chamelons. 


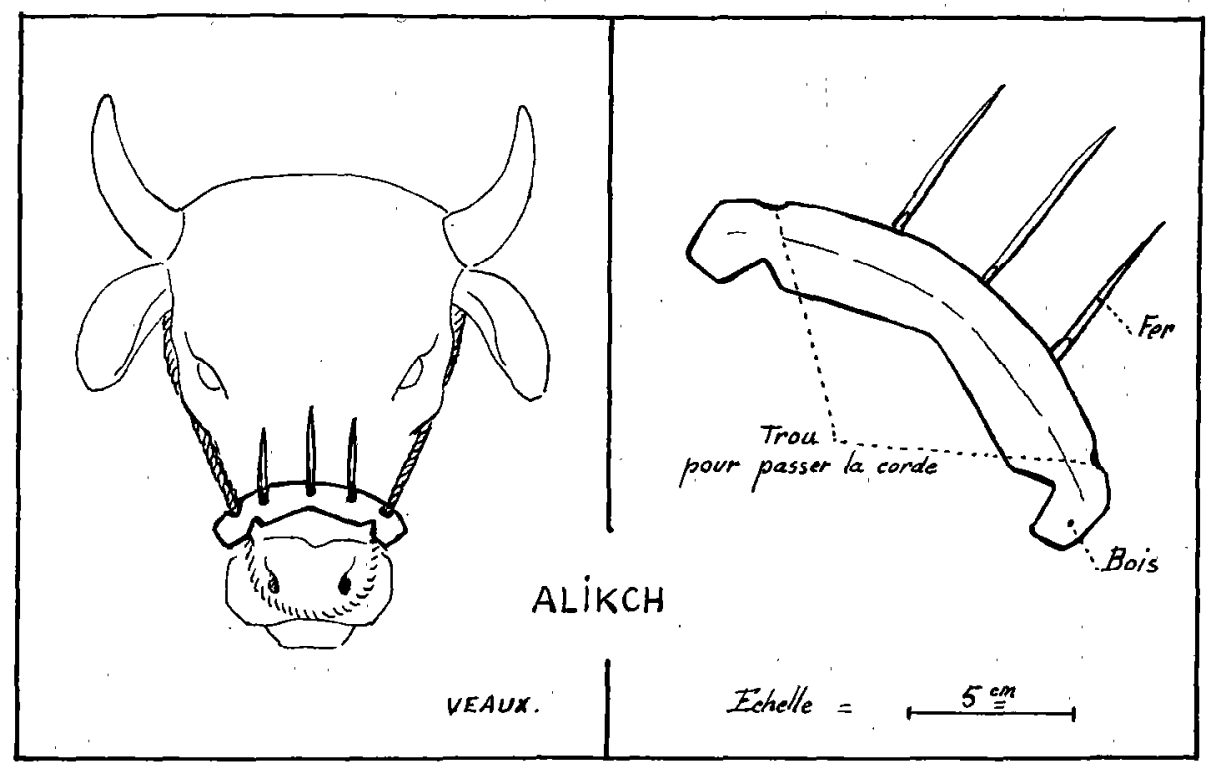

Figure 1.

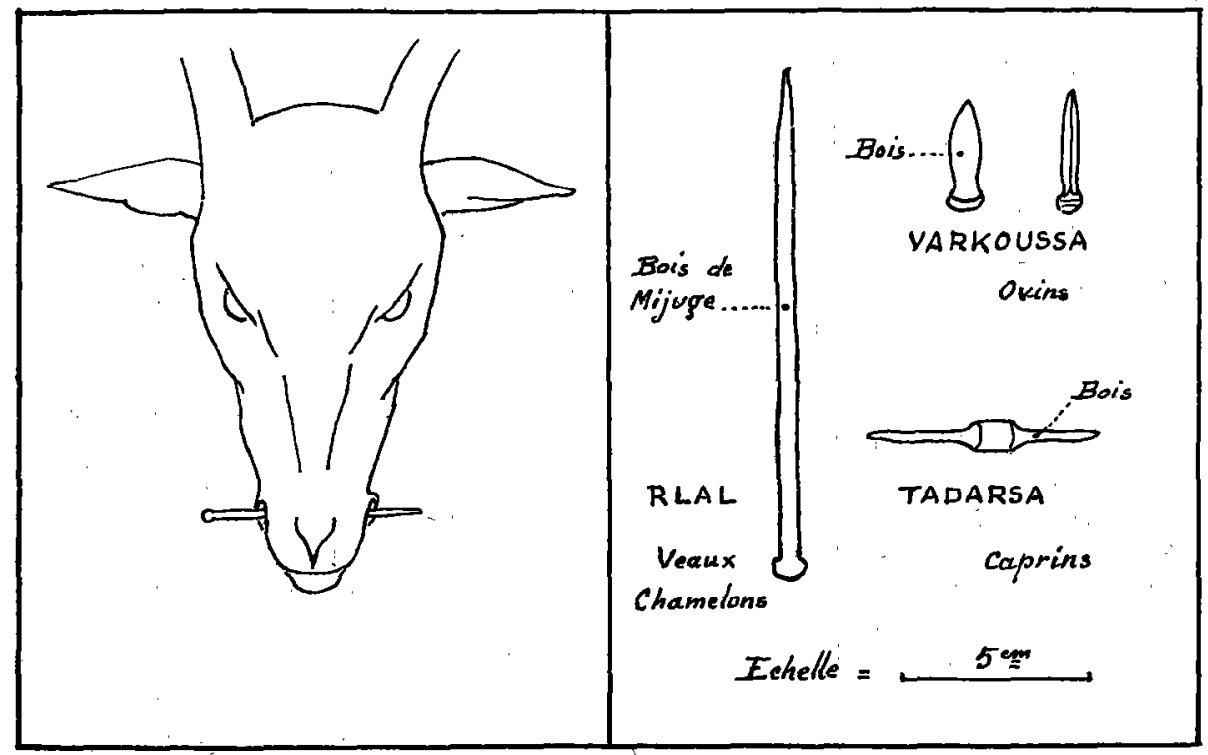

Figure 2. 


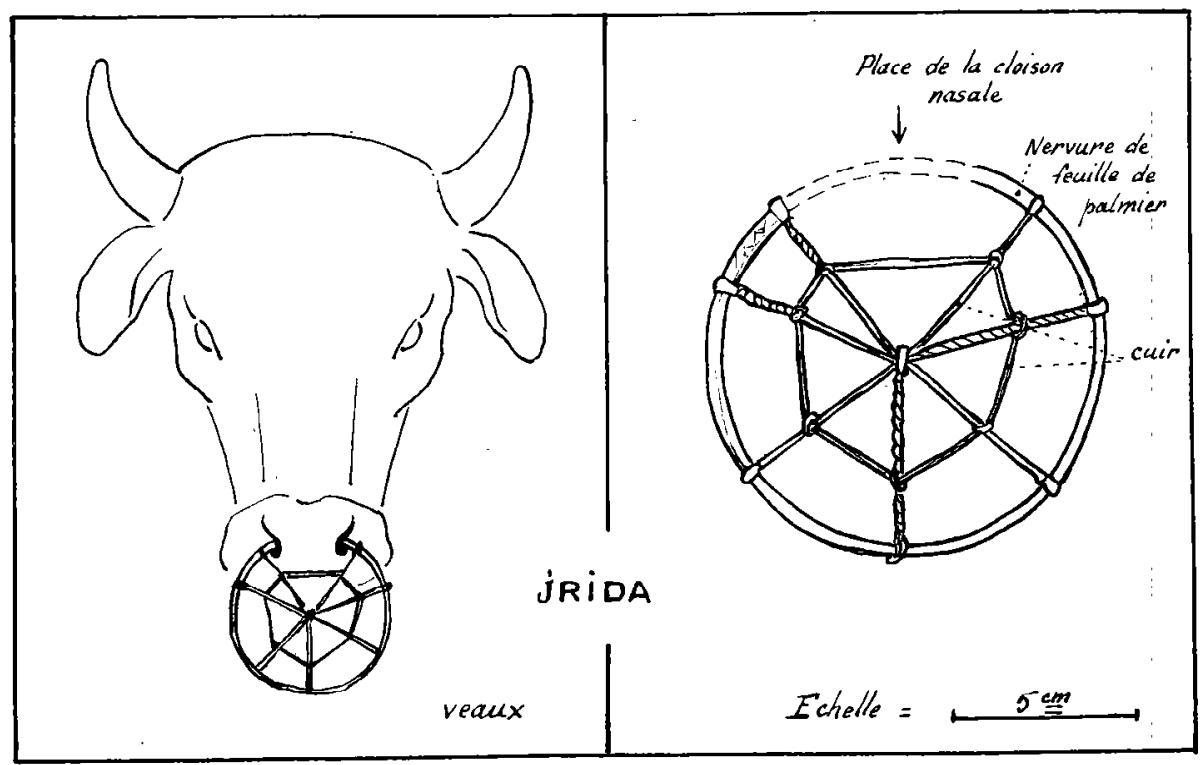

Figure 3.

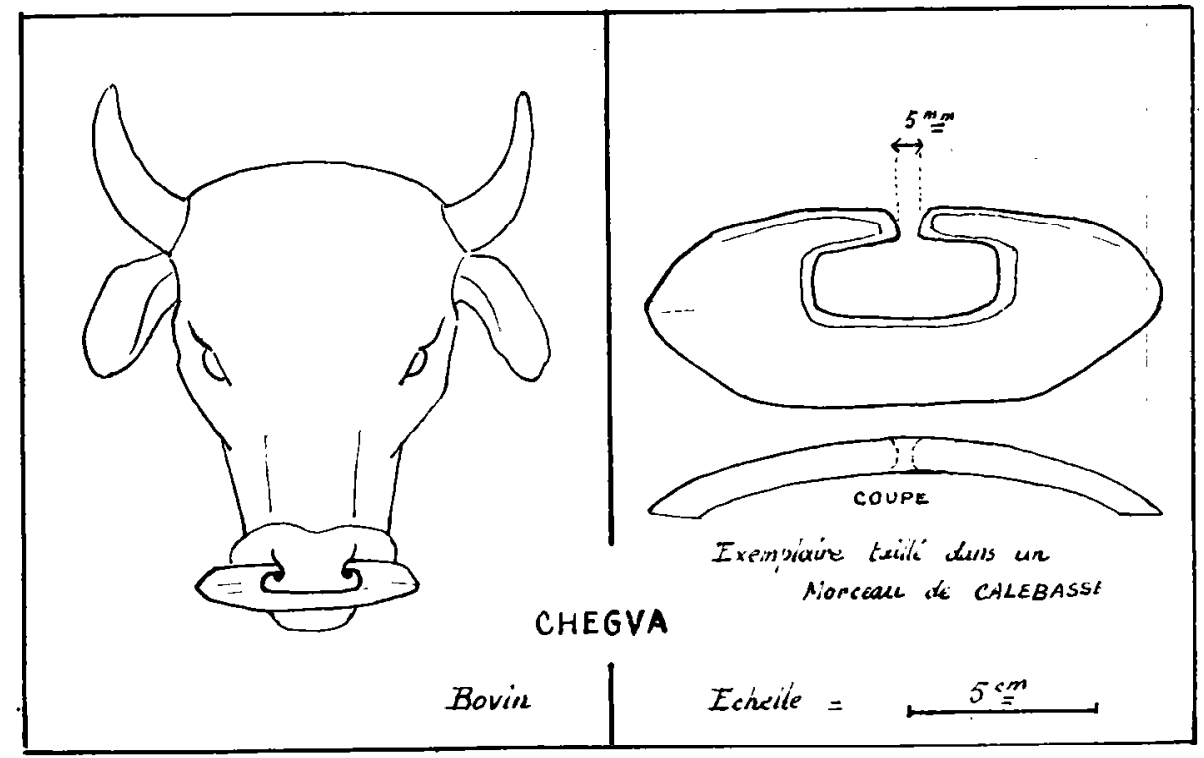

Figure 4. 


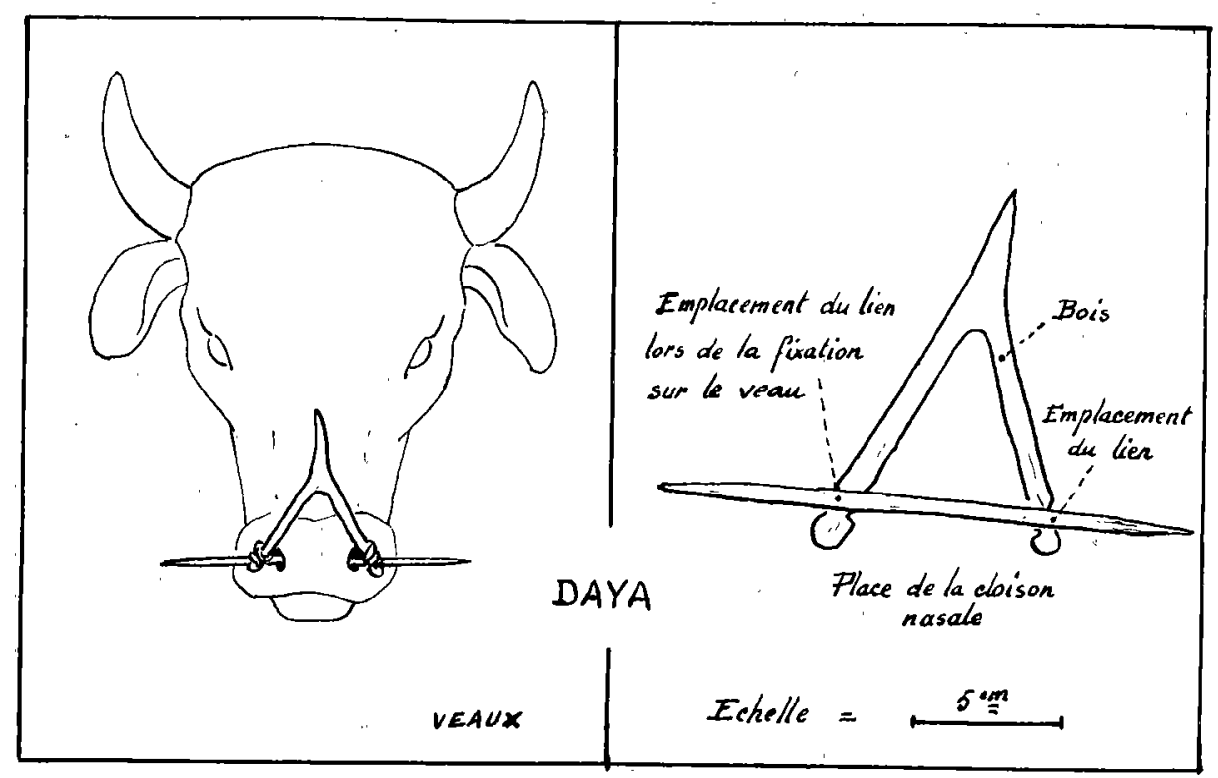

Figure 5.

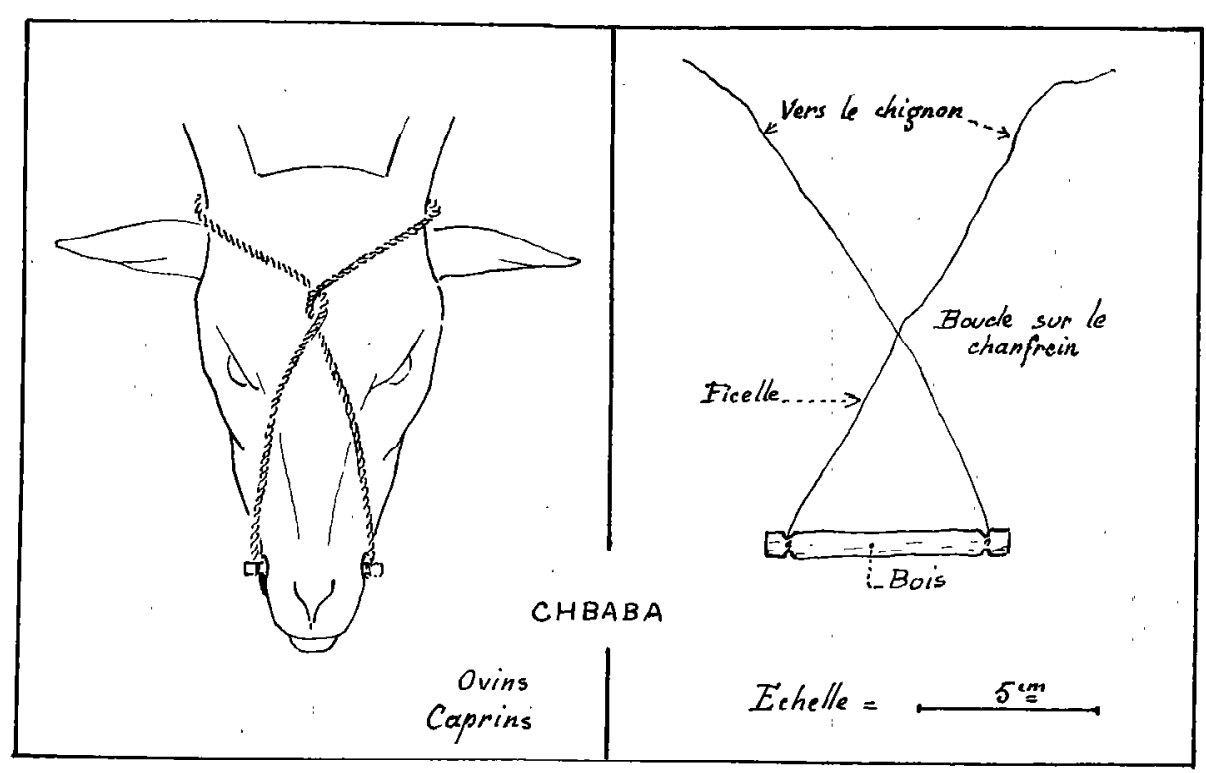

Figure 6. 
Elle consiste à inciser la peau du chanfrein de façon à délimiter une languette de peau que l'on récline vers les naseaux, que l'on enroule et qu'on serre à la base par une ficelle. On laisse celle-ci en place pendant deux jours. Pour que la cicatrisation soit moins rapide, certains éleveurs mettent même du sable ou des morceaux de paille dans la plaie; d'autres fendent le morceau de peau au bout de quinze jours.

Malgré tous ces raffinements cette méthode est assez peu efficace.

b) Procédés avec fixation d'appareil défensif.

Les pasteurs maures ont imaginé divers instruments qui portent les noms suivants : «alikch», 《 rlal », « jrida », « daya », « chegva », « tadersa », "verkoussa », « chbâba ». Ces instruments sont confectionnés, selon le cas, avec du bois d' «adress» (1) (alikch), du bois de «mijuge » (2) (rlal), du fer (alikch), des nervures de feuilles de palmiers (jrida), de la ficelle et du cuir. Les forgerons maures fabriquent aisément ces appareils très simples et les bergers taillent souvent eux-mêmes les petits instruments en bois destinés aux agneaux et aux chevreaux, comme le «tadersa» ou le « verkoussa ».

Tous ces appareils peuvent se classer en trois groupes d'après leur mode de fixation :

10 Ceux qui sont fixés par piqûre d'une partie molle de la tête (cartilage internasal, aile de la narine, joue, palais) :

a) appareils traversant la cloison médiane du nez : " jrida », « daya »;

b) appareil traversant la narine : " rlal » (consolidé par un lien);

c) appareil traversant les parois buccales près des commissures des lèvres : «tadersa ».

d) appareil planté dans le sillon médian du palais: « verkoussa ».

$2^{\circ}$ Ceux qui sont maintenus par des liens passant sur la nuque ou fixés aux cornes :

a) appareil placé transversalement dans la bouche et maintenu par des cordelettes nouées sur le chignon ou les comes : " chbâba »;

b) appareil posé sur le chanfrein et maintenu de la même façon par des liens : « alikch ".

$3^{\circ}$ Ceux qui, comme le "chegva», sont simplement suspendus au mufle grâce à une encochè dans laquelle s'engage la cloison internasale.

Les croquis représentant ces différents appareils permettront d'en mieux comprendre la structure et la mise en place. Quant à leur fonctionnement, il est aisé à deviner : ces appareils agissent soit sur

(1) Commiphora africana.

(2) Grewia bicolor. la mère en lui infligeant des piqûres à la mamelle, à la cuisse ou au ventre (alikch, rlal, daya), soit sur le jeune en rendant la succion impossible, par barrage de la bouche (jrida, chegva), par appui sur la langue (chbâba, tadersa), par douleur au palais (verkoussa). Certains de ces appareils agissent d'ailleurs de façon mixte (tadersa). Leur emploi suivant les espèces peut être résumé dans le tableau suivant :

\begin{tabular}{|c|c|c|c|c|c|}
\hline & 总 & 总 & 空 & 敫 & $\begin{array}{l}\text { CAME- } \\
\text { LIDÉS }\end{array}$ \\
\hline Jrida ... & + & 一 & - & $\div$ & $\div$ \\
\hline Daya & + & 一 & - & $i$ & - \\
\hline Rlal ..... & + & - & - & - & + \\
\hline Verkoussa & 一 & + & 一 & $\frac{1}{i}$ & 一 \\
\hline Tadersa & - & - & + & $\frac{1}{\vdots}$ & 一 \\
\hline Chbâba. & - & + & + & : & - \\
\hline Alikch & + & 一 & - & + & - \\
\hline Chegva. & + & 一 & 一 & - & - \\
\hline
\end{tabular}

Il peut toutefois être utile de préciser quelques détails :

Alikch : Chez les veaux, est employé sur des animaux d'un an que les Maures préfèrent ne pas laisser au campement parce qu'ils jettent le trouble parmi les animaux plus jeunes. Le soir, lorsque le troupeau est rentré, on débarrasse le veau de son alikch, on le laisse un peu téter puis on procède à la traite.

Chez l'ânon on laisse l'alikch en place pendant 2 ou 3 jours lorsqu'on veut sevrer définitivement l'animal. Si cela ne suffit pas on lui fend les narines.

Jrida : l'appareil est oscillant, aussi le veau parvient-il à brouter mais lorsqu'il relève la tête pour téter le jrida bascule et se place devant la bouche.

Chegra : fonctionne de la même façon que le précédent. Il se pourrait qu'il soit d'origine peule; en effet les peuls emploient un appareil identique appelé "lalogal » (mot dérivé de "lahal » : calebasse; cet appareil est en effet fabriqué en général à l'aide d'un morceau de calebasse).

Verkoussa : il faut préciser que cet instrument est destiné à être logé dans la fente palatine des agneaux mais non dans celle des chevreaux parce que, disent les pasteurs, ces derniers ont une fente palatine trop large et que « verkoussa leur coupe la respiration ». L'instrument est souvent laissé en 
place pendant toute la vie de l'animal; il arrive ainsi qu'on le retrouve sur des animaux de 6 ans. Parfois le berger l'enlève au bout d'un certain temps ou bien il tombe tout seul.

Tadersa : les pasteurs prétendent qu'ils n'utilisent pas cet instrument chez les caprins parce que ceux-ci ont une bouche moins extensible que celle des ovins.

Pour bien comprendre les diverses modalités d'utilisation de ces. différents appareils, il faut se rappeler que, chez les Maures, les bovins sont placés au pâturage sans gardien tandis que les petits ruminants sont surveillés par des bergers. Il faut aussi tenir compte du fait que, le plus souvent, les vaches ne se laissent traire que si l'on permet aux veaux de téter un peu. Au contraire la traite peut habituellement être effectuée cher les brebis et les chèvres en l'absence de leurs petits. Enfin, l'âge du jeune animal intervient aussi dans le choix du moyen de sevrage.

Ainsi le veau ayant moins d'un an, facile à surveiller, reste au campement pendant le jour; il est attaché pendant la nuit. On ne lui met jamais d'appareil du genre alikch ou autre.

Le veau ayant plus d'un an, très difficile à surveiller, doit accompagner sa mère au pâturage. Deux cas se présentent alors : si la mère n'est pas en gestation, son veau reçoit un appareil de sevrage provisoire (alikch). Celui-ci est retiré chaque soir au moment de la tétée et de la traite. Si la mère est en gestation, on sèvre définitivement le veau en lui imposant un appareil du type jrida, daya ou rlal, et l'on ne pratique pas de traite.

Chez les petits ruminants, les procédés varient aussi selon l'âge des jeunes. Les agneaux ayant moins de 15 jours sont mis à l'attache ou en zériba dans la journée; ils passent la nuit avec leurs mères qu'on ne trait pas. Ceux qui ont 15 à 45 jours sont isolés de même pendant le jour; ils peuvent téter le soir après la traite et restent toute la nuit avec leurs mères. De 2 mois à 2 mois et demi environ, ils sont également séparés des brebis dans la journée, peuvent téter une seule fois dans la soirée, après la traite, puis sont attachés pour la nuit. Au cours du $3^{\circ}$ mois les agneaux commencent à suivre le troupeau au pâturage : deux procédés de sevrage sont alors utilisés: ou bien la ligature des trayons des mères ou bien l'échange des agneaux de deux troupeaux différents. Le sevrage définitif est obtenu pour les animaux âgés de 3 à 4 mois, par application de l'un des instruments décrits ci-dessus (verkoussa ou tadersa).

Chez les ânes, le sevrage se fait en général naturellement, la sécrétion lactée se tarissant chez la mère au bout de 6 à 7 mois. Mais il arrive qu'on veuille sevrer un ânon de 4 ou 5 mois; on utilise alors l'alikch qu'on laisse d'abord en place pendant une journée seulement pour voir si l'ânon peut rẻellement se passer de lait; si oui on replace l'appareil et, petit à petit, la mère chasse le jeune. On peut aussi pratiquer la fente des narines de l'ânon.

Les procédés utilisés pour sevrer les poulains sont bien différents. En effet, bien que l'élevage des chevaux soit assez peu répandu en Mauritanie (sauf dans certaines tribus du sud du Brakna), les Maures, qui considèrent le cheval comme un animal noble, n'utilisent pas les procédés brutaux précédemment décrits lorsqu'ils doivent sevrer les poulains.

Pendant les 4 jours qui suivent la naissance d'un poulain, c'est sa mère qui est attachée' et non lui; matin et soir on apporte à la jument une boisson chaude faitc avcc de la farine de mil. Le jeune tète comme il lui plaît. Du $4^{\circ}$ jour jusqu'au sevrage, le poulain est mis à l'attache en permanence; la mère, remise en liberté, va paître à sa guise et revient quand elle le veut pour donner à boire au jeune. Le sevrage a lieu entre 3 et 4 mois. On remet la jument à l'attache, on la trait une fois par jour pendant 2 à 3 jours en faisant à chaque fois des applications d'eau froide sur la mamelle; la sécrétion lactée se tarit rapidement.

Lorsque les Maures veulent que le poulain continue à bénéficier du régime lacté, ils lui attribuent comrne nourrices une ou deux vaches ou une chamelle. Certains éleveurs riches mettent même à la disposition du poulain le lait produit par plusieurs brebis. Le berger trait celles-ci au pâturage et donne le lait au poulain de, sorte que l'on peut voir des chevaux adultes qui continuent, par habitude, à aller au pâturage avec les moutons.

L'éleveur maure qui veut obtenir le plus grand nombre possible de poulains procede toujours au sevrage précoce de façon à faire saillir à nouveau la jument le plus tôt possible:

\section{Comparaison des procédés maures et des procédés peuls.}

Nous passerons rapidement en revue les procédés de sevrage utilisés par les Peuls en n'insistant que sur ceux qui présentent quelque particularité remarquable, et en suivant le même plan que précédemment.

I. - Les procédés par séparation. Chez les Peuls, les veaux de moins de 6 mois sont rarement attachés mais le plus souvent enfermés dans un enclos (《oudoum»). Toutefois, pour séparer les veaux d'une semaine des animaux plus âgés, les pasteurs peuls attachent les premiers à l'aide d'une corde 


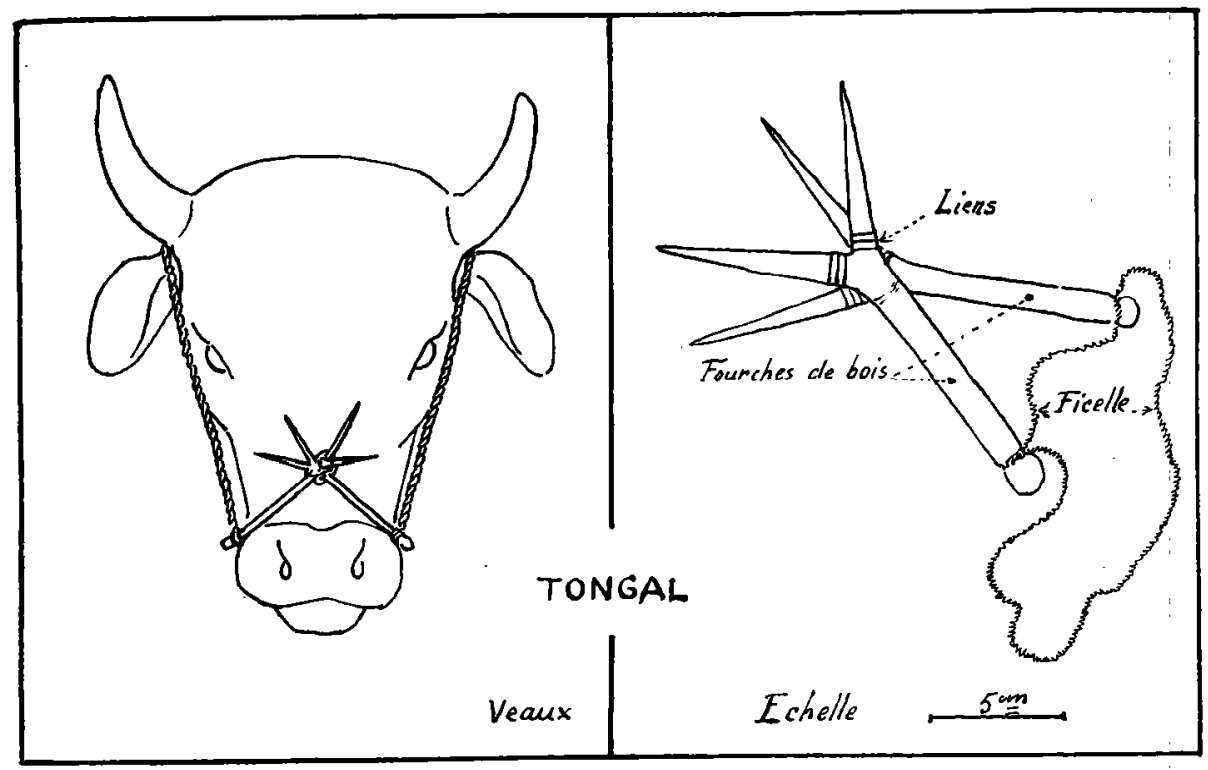

Figure 7.

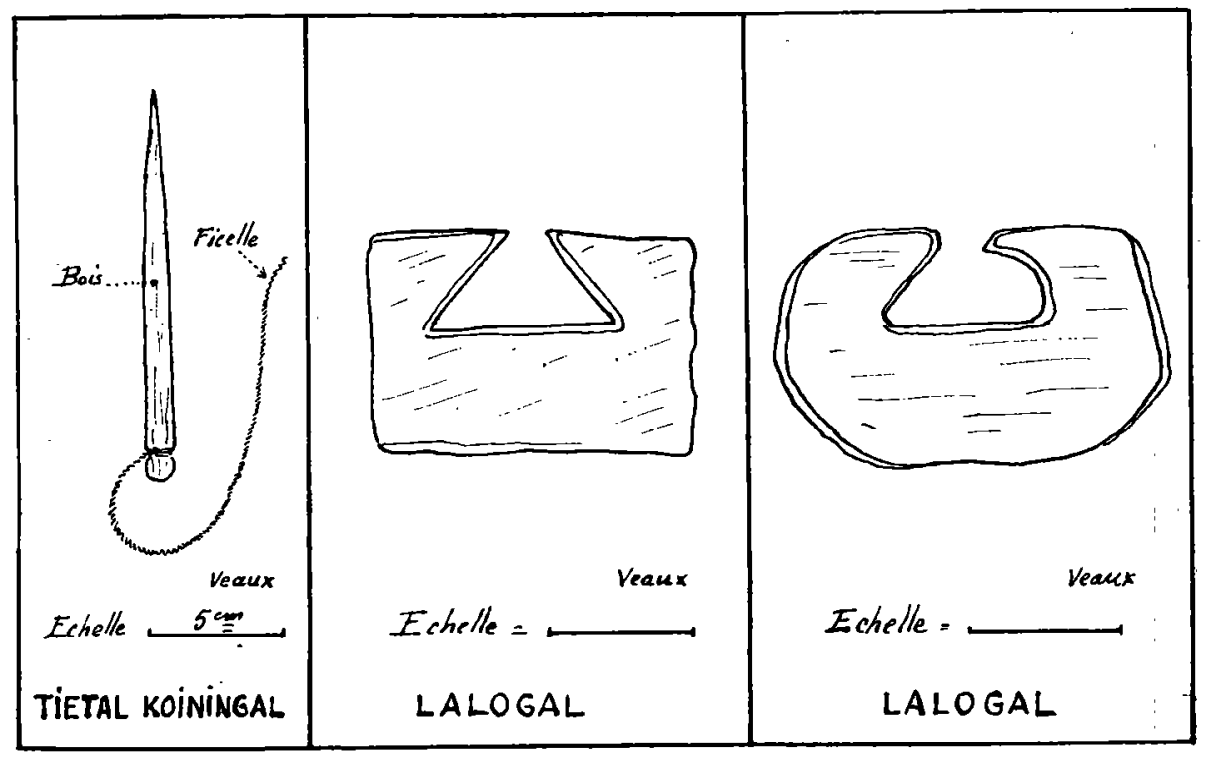

Figure 8. 
fixée à la patte. Les petits ruminants sont en général parqués jusqu'à l'âge de 3 mois dans un enclos d'épineux (" oudoum-bourti »: parc des agneaux).

II. - Les procédés mettant én œeuvre des appareils fixés aux rnamelles ne sont pas utilisés.

III. - Les procédés impliquant une intervention sanglante au niveau du mufle du jeune sans fixation d'appareil, ne sont pas employés non plus.

IV. - Appareils fixés sur le jeune.

Ils portent les noms de : «tongal» (pluriel : tondié); «tiétal palingal », « tiétal koiningal » (pluriel : tiété); « lalogal » (pluriel laladé); « kel- équivalent maure en fer qui nécessite l'emploi du feu.

Tiétal koiningal : ou tiétal vertical correspond au rlal maure. Comme lui il est le plus souvent fixé à la narine mais parfois aussi à la lèvre supérieure.

Tiétal palingal ou tiétal horizontal : traverse la cloison nasale du veau, comme la partie horizontale du daya maure, mais il en differe par l'absence de la pièce de bois en Y qui est remplacée par un lien de cuir et une ficelle passant sur le chanfrein et servant uniquement à la fixation. En compen-

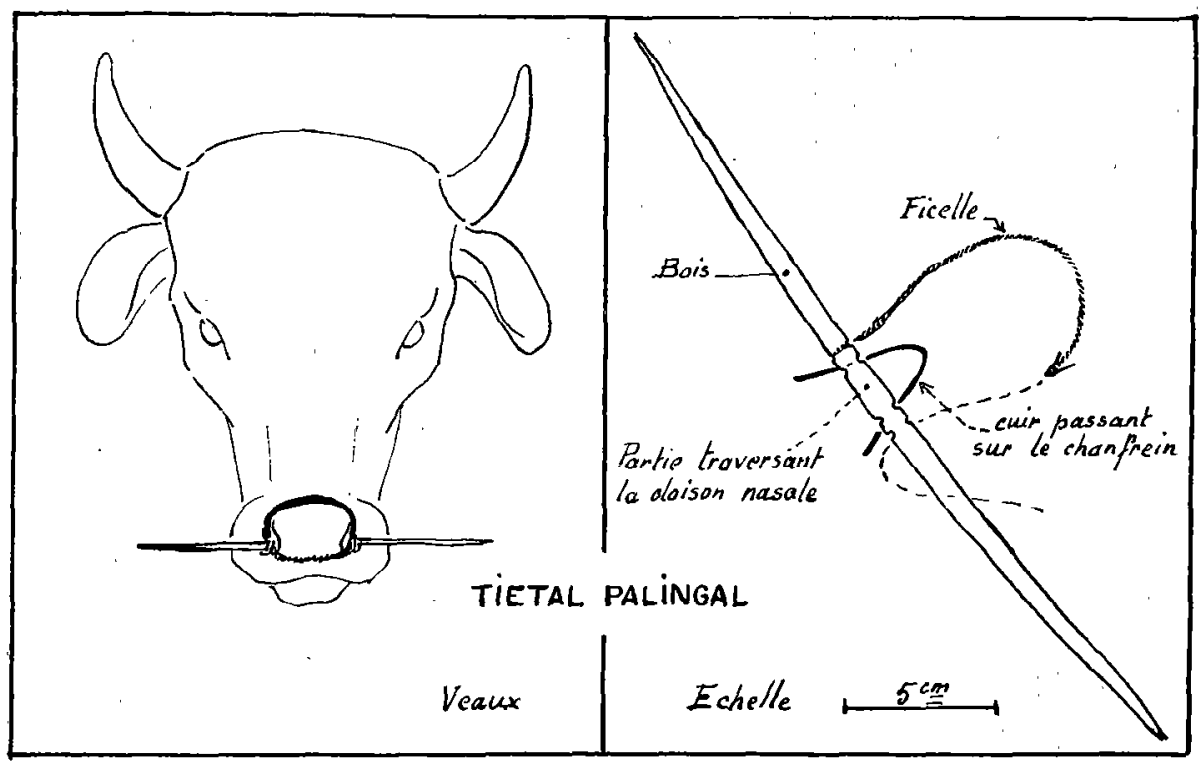

Figure 9.

boungol» (pluriel : kelbouli); « tienguélel ». Les croquis qui les représentent seront plus explicites qu'une longue description. Les Peuls utilisent pour les confectionner du bois, surtout de «keli» (1), des fragments de l'enveloppe d'une pastèque appelée «horde », de la ficelle, et du cuir; le fer n'est pas employé.

Des analogies très grandes existent entre ces appareils peuls et les instruments maures.

Tongal : correspond, à l'alikch maure. Le but recherché et le mode de fixation sont identiques. On remarquera que l'instrument peul, constitué uniquement de deux fourches de bois et de quelques liens, est beaucoup plus facile à fabriquer que son

(1) Grewia bicolor (Tiliacées). sation, peut-on dire, la longueur du tiétal est presque le double de celle du daya.

Lalogal : nous avons déjà dit qu'il est identique au chegva maure. Le plus souvent le lalogal, fabriqué dans un fragment de péricarpe de pastèque, est de forme rectangulaire avec une encoche triangulaire; mais il existe aussi des laladé de forme arrondie.

Kelboungol : ne diffère guère du chbâba maure et s'emploie de la même façon chez les petits ruminants.

Tienguelel : c'est le jrida maure, qu'utilisent d'ailleurs les bergers maures au service des Peuls. Le mot tienguelel semble un diminutif de tiengal qui désigne chez les Peuls une sorte de panier servant à récolter le mil. 
L'emploi de ces divers appareils, selon l'espèce du jeune animal à sevrer, peut être résumé dans le tableau suivant :

\begin{tabular}{|c|c|c|}
\hline & BOVINS & OVINS-CAPRINS \\
\hline Tongal ............ & + & - \\
\hline Tiétal palingal & + & - \\
\hline Tiétal koiningal. & + & - \\
\hline Lalogal. . & + & - \\
\hline Tienguélel & + & 一 \\
\hline Kelboungol & — & + \\
\hline
\end{tabular}

On constatera qu'ils ne sont appliqués ni aux poulains, pour lesquels les procédés utilisés sont les mêmes que chez les Maures, ni aux ânons dont on laisse le sevrage s'effectuer naturellement.

Les moyens successivement mis en cuvre par les Peuls pour limiter la consommation de lait des jeunes veaux sont: la mise à l'attache pour les animaux de moins d'une scmaine, la mise on "oudoum» ensuite, jusqu'à l'âge de 6 mois. Les veaux ayant plus de 6 mois sont munis d'un tongal. Pour les sevrer définitivement, entre 6 mois et $I$ an, on utilise le tiétal ou le lalogal. Pendant les 6 mois qui suivent le sevrage, le veau est nommé « hodangé » (pluriel: kodadi).

Pour les petits ruminants, les Peuls procèdent en général comme les Maures, à quelques différences près. Ainsi les agneaux sont attachés pendant la nuit dès qu'ils atteignent l'âge de 1 mois; les bergers les font téter surtout le matin. En vue d'obtenir plus tard de beaux animaux destinés à la reproduction ou, après castration, à la boucherie, les bergers laissent certains agneaux téter à discrétion. En général le sevrage est réalisé, lorsque les animaux ont 3 mois, par pose d'un kelboungol; les agneaux sont alors nommés «kourouri » (singulier : kourourol) pendant $l$ an; le même mot sert pour les chevreaux sevrés.

Au terme de cette étude, effectuée dans la région du Brakna et qui n'a pas la prétention de présenter un tableau complet des procédés de sevrage utilisés dans toute la Mauritanie, il semble toutefois possible d'affirmer que les méthodes maures sont plus variées, plus ingénieuses, mais aussi plus sanglantes que celles des Peuls. En effet, alors que les Peuls semblent avoir porté leurs efforts surtout dans la recherche d'instruments pour le sevrage des bovins (ils ne disposent que d'un seul instrument pour les petits ruminants), les Maures, qui ont éléndu 1'application de leurs méthodes au chameau et à l'âne, ont aussi trouvé de plus nombreux procédés utilisables chez les ovins et caprins.

Quelques petits problèmes, d'intérêt ethnographique, peuvent également être posés. Y a-t-il une filiation, et laquelle, entre les procédés peuls et maures? L'alikch est-il un dérivé du tongal, le daya une complication du tiétal ? Le kelboungol est-il un chbâba modifié ou inversement?

On peut aussi penser qu'il serait intéressant de comparer les methodes décrites ici avec celles qu'utilisent d'autres éleveurs nomades, non seulement d'A.O.F. mais de diverses autres contrées.

Enfin, du point de vue zootechnique, il serait peut-être fructueux d'envisager les retentissements que la stricte limitation de l'alimentation lactée ne peut manquer d'avoir sur l'organisme des jeunes animaux, les facteurs qui permettent à ces derniers de s'adapter plus ou moins vite et plus ou moins bien à une alimentation d'adultes, les conséquences de cette sélection naturelle et son éventuelle importance pour l'amelioration de l'élevage mauritanien. 